\title{
Transoral Trans-Stomal Microdebrider Excision of Tracheal Papillomatosis
}

Marc C. Thorne, MD; Karen B. Zur, MD

Objectives: To describe a technique for surgical removal of recurrent respiratory papilloma in the distal trachea in patients with an indwelling tracheostomy tube.

Methods: A transoral trans-stomal technique for removal of distal tracheal papilloma using a modified ventilating bronchoscope setup and a microdebrider with a rotatable Skimmer blade (XOMED Products, Jacksonville, Florida, USA).

Results: Surgical removal of papillomas in the distal trachea is a challenging procedure, involving potentially competing demands for visualization, instrumentation, and ventilation. Previously described methods for management of these challenging cases provide limited ability to deliver continuous oxygenation and ventilation during the procedure. The transoral trans-stomal technique overcomes these limitations in patients who have an existing tracheostomy.

Conclusions: This technique allows for precise removal of papilloma in the distal trachea, while providing for excellent visualization and continuous oxygenation and ventilation of the patient.

Key Words: Recurrent respiratory papillomatosis, papilloma, laryngeal neoplasms, tracheostomy.

Laryngoscope, 119:964-966, 2009

\section{INTRODUCTION}

Recurrent respiratory papillomatosis $(\mathrm{RRP})$ is a virally mediated disease resulting in exophytic lesions of the airway. The lack of curative therapy, tendency for recurrence and spread, and variable natural history make treatment of this disease difficult. Despite investigation of a number of medical and surgical therapies, repeated

From the Department of Otolaryngology, Division of Pediatric Otolaryngology, University of Michigan, Ann Arbor, Michigan, U.S.A (м.с.т.); and the Division of Otolaryngology, Children's Hospital of Philadelphia, Department of Otorhinolaryngology-Head and Neck Surgery, University of Pennsylvania School of Medicine, Philadelphia, Pennsylvania, U.S.A. (к.B.z.).

Editor's Note: This Manuscript was received on August 8, 2008 and accepted for publication October 16, 2008.

Presented as a poster at the 88th Annual American BronchoEsophagological Association Annual Meeting, Orlando, Florida, U.S.A., May 1-2, 2008.

No relevant funding support.

Send correspondence to Marc C. Thorne, MD, F6866 Mott, SPC 5241, 1500 East Medical Center Drive, Ann Arbor, MI 48109-5241. E-mail: mthorne@med.umich.edu

DOI: 10.1002/lary.20163 surgical debulking remains the mainstay of treatment. Although a variety of methods for surgical removal have been described, a microdebrider technique was recently demonstrated to be the preferred method for surgical therapy among American Society of Pediatric Otolaryngology members. ${ }^{1}$

The larynx is the most commonly involved anatomic site, with estimates of involvement of the lower airways ranging from $5 \%$ to $29 \%$. Although a benign disease, distal spread portends a poor prognosis, with mortality rates for pulmonary papillomatosis on the order of $50 \%{ }^{2}$ In addition, distal spread of disease also presents difficulties for surgical removal due to difficulty in accessing the distal airway in cases of laryngeal obstruction and proliferative tracheal disease.

The role of tracheotomy in the management and spread of distal RRP is somewhat controversial. Distal papillomas have been noted to develop in greater than $50 \%$ of patients requiring a tracheotomy for management of their RRP. However, it has also been noted that patients requiring tracheotomy for RRP more often present at a younger age and with distal disease prior to their tracheotomy. Whether tracheotomy increases the risk of distal spread or is a marker of individuals with severe disease is unclear. ${ }^{3}$

We describe our technique for removal of tracheal and bronchial papillomas in patients who have required a tracheotomy for airway maintenance. This technique has been used extensively on a patient referred to the senior author (KBZ) after having undergone urgent tracheotomy for airway obstruction at an outside institution. She has since developed pulmonary involvement, and has been managed with bimonthly surgical debridement in order to maintain a patent airway. In addition, she has received adjuvant therapy including a protocol of intravenous cidofovir, and more recently with subcutaneous interferon alpha. The typical appearance of her laryngeal and tracheal disease can be seen in Figure 1. This technique allows for maintenance of the airway and continuous ventilation as well as excellent visualization for surgical removal of distal disease.

\section{OPERATIVE TECHNIQUE}

In preparation for the procedure, a surgical table is arranged with all required instrumentation (see Fig. 2). 


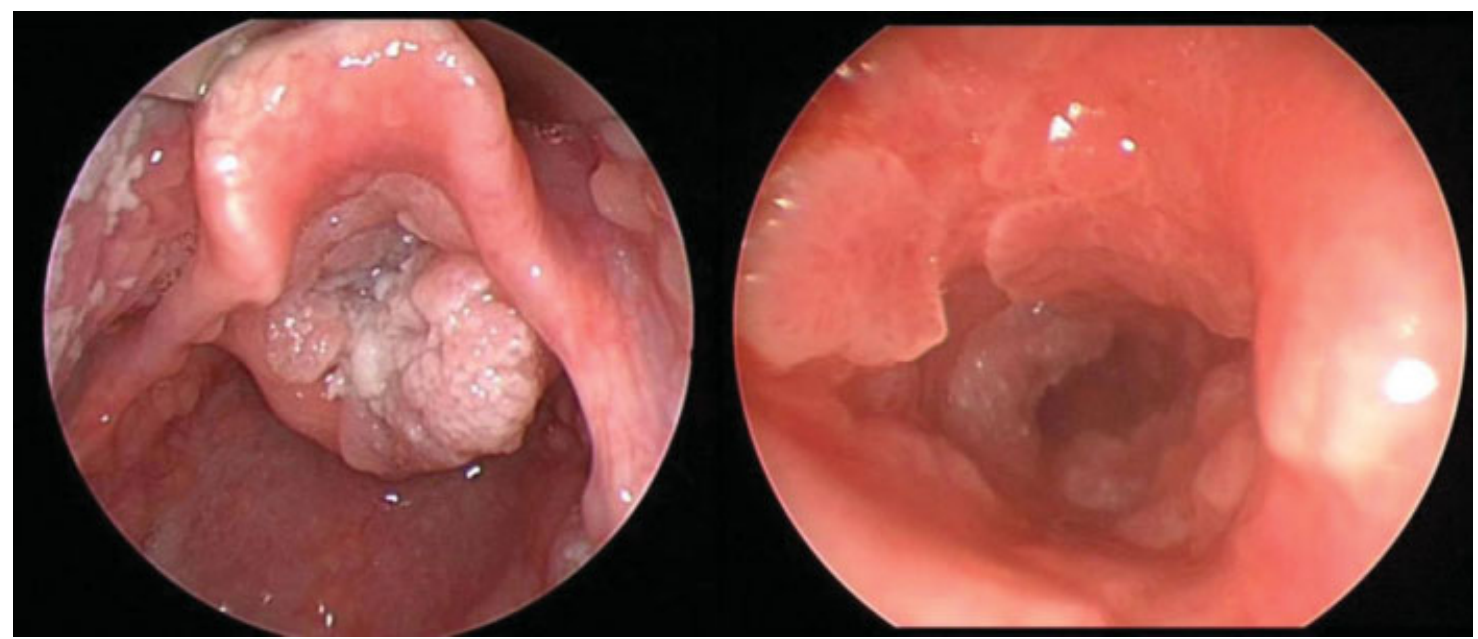

Fig. 1. On left, typical appearance of obstructing laryngeal papillomas at time of bimonthly surgical debridement. On right, typical appearance of tracheal disease at time of bimonthly surgical debridement. Note diffuse tracheal involvement with carina visible distally. [Color figure can be viewed in the online issue, which is available at www.interscience.wiley.com.]

This includes standard instrumentation for microlaryngoscopy and bronchoscopy. In addition, pledgets soaked in oxymetazoline are available for assistance with hemostasis. The standard set-up for the bronchoscope is modified as follows. The locking coupling for the telescope and the bronchoscope is replaced with the spacer typically used for esophagoscopy. This allows the telescope to sit slightly recessed within the bronchoscope, helping to prevent blood and secretions from contaminating the telescope and impairing visualization. A flexible suction catheter is placed through the side arm of the bronchoscope to assist in removal of blood from the field (see Fig. 3).

Anesthesia is induced through the patient's existing tracheotomy tube. Under suspension laryngoscopy, the obstructing laryngeal disease is first addressed in order to provide a natural airway and to permit access to the subglottis and trachea. With this access established, a two-surgeon technique utilizing one bronchoscopist and

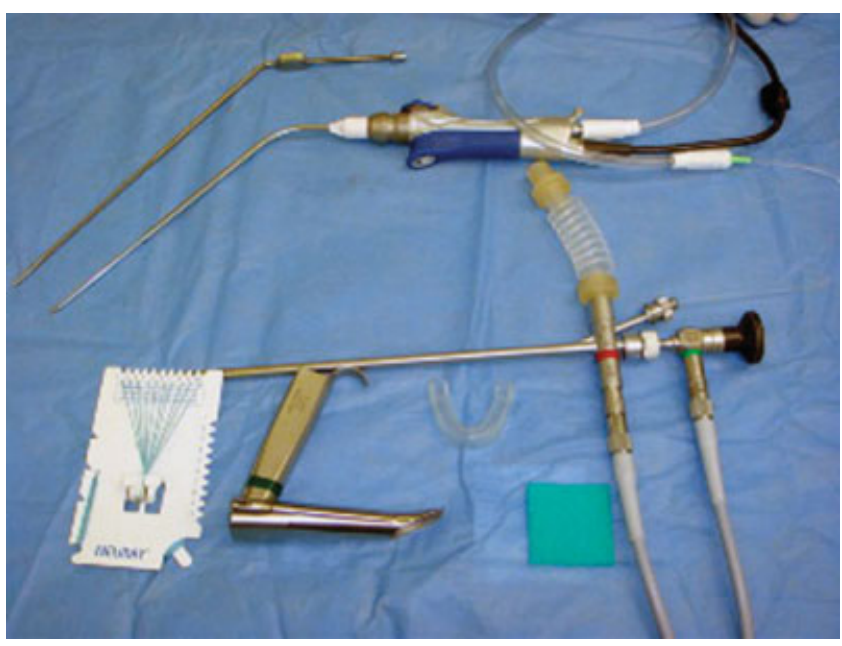

Fig. 2. Set-up of operative table including standard instrumentation for microlaryngoscopy and bronchoscopy. [Color figure can be viewed in the online issue, which is available at www.interscience. wiley.com.] one operative surgeon is utilized to assess the tracheal disease. The ventilating bronchoscope with suction and the recessed telescope is then passed into the trachea by the bronchoscopist, allowing for simultaneous ventilation and visualization as the tracheotomy tube is removed. The operative surgeon then passes a microdebrider with a rotatable Skimmer blade (XOMED Products, Jacksonville, FL) through the stoma. Resection of tracheal and bronchial papillomas can then be performed under ideal visualization while allowing continuous ventilation of the patient (see Fig. 4). Maintenance of suspension laryngoscopy throughout the procedure allows for efficient cleaning of the telescope should visualization become obscured by blood or secretions.

\section{DISCUSSION}

Surgical removal of papillomas in the distal trachea presents several challenges, including potentially

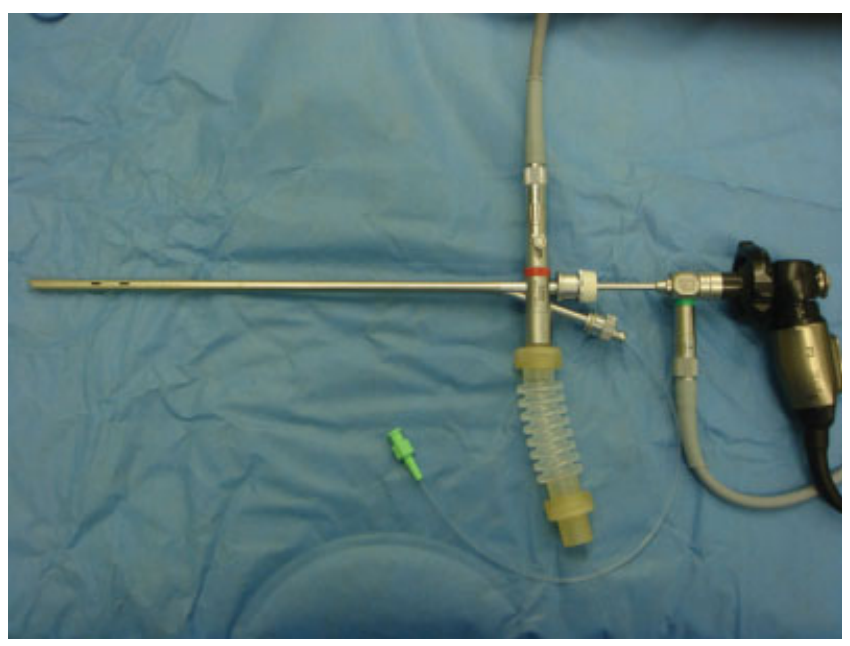

Fig. 3. Modified setup for bronchoscope including spacer to recess telescope and flexible suction catheter. [Color figure can be viewed in the online issue, which is available at www.interscience. wiley.com.] 


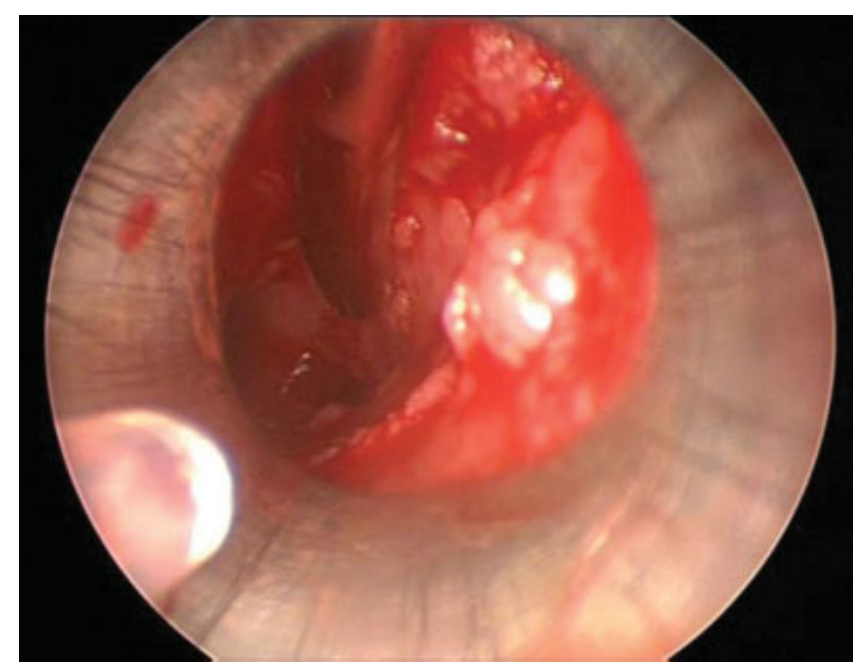

Fig. 4. Intraoperative telescopic view of microdebrider introduced through the stoma. Note recessed telescope and flexible suction at lower left. [Color figure can be viewed in the online issue, which is available at www.interscience.wiley.com.]

competing demands for visualization, instrumentation, and ventilation. The ideal technique would allow excellent visualization for precise removal of disease while limiting collateral injury. In addition, ventilation of the patient should not be compromised.

Rees et al. describe use of the microdebrider for removal of tracheal papilloma in conjunction with visualization by a rigid telescope. They note that the small confines of the pediatric larynx do not allow simultaneous use of a ventilating bronchoscope and the microdebrider in the trachea. ${ }^{4}$ This technique does not allow for continuous ventilation of the patient, but is clearly of great utility in patients with distal disease in whom tracheotomy has not been required. Ulualp et al. describe a technique for trans-stomal management of tracheal papillomas where the bronchoscope and microdebrider are both introduced through the patient's stoma. However, the size of the ventilating bronchoscope is limited to 2.5 due to the constraints of stoma size. ${ }^{5}$ Ventilation through a small 2.5 bronchoscope may be ineffective, especially in an older child.

Although the role of tracheotomy in promoting distal spread of disease remains somewhat uncertain, we do not advocate tracheotomy in order to facilitate use of this technique. However, in cases where a tracheotomy has been required and the laryngeal inlet size limits placement of multiple instruments, we believe this technique offers advantages over those previously described. These advantages include the ability to control the airway and provide for continuous ventilation, as well as excellent visualization and access for controlled removal of disease. In contrast to techniques using laser technology, the risk of airway fire is avoided, as is the theoretical risk of spread of virus through laser plume. Disadvantages include the dependence on an existing tracheostomy, need for two surgeons, and lack of hemostasis with the microdebrider.

\section{BIBLIOGRAPHY}

1. Schraff S, Derkay CS, Burke B, Lawson L. American Society of Pediatric Otolaryngology members' experience with recurrent respiratory papillomatosis and the use of adjuvant therapy. Arch Otolaryngol Head Neck Surg 2004; 130:1039-1042.

2. Soldatski IL, Onufrieva EK, Steklov AM, Schepin NV. Tracheal, bronchial, and pulmonary papillomatosis in children. Laryngoscope 2005;115:1848-1854.

3. Derkay CS, Darrow DH. Recurrent respiratory papillomatosis. Ann Otol Rhinol Laryngol 2006;115:1-11.

4. Rees CJ, Tridico TI, Kirse DJ. Expanding applications for the microdebrider in pediatric endoscopic airway surgery. Otolaryngol Head Neck Surg 2005;133:509-513.

5. Ulualp SO, Ryan MW, Wright ST. Microdebrider removal of tracheal papilloma via tracheostomy in the child with an obliterated larynx. J Laryngol Otol 2007;121:1070-1072. 